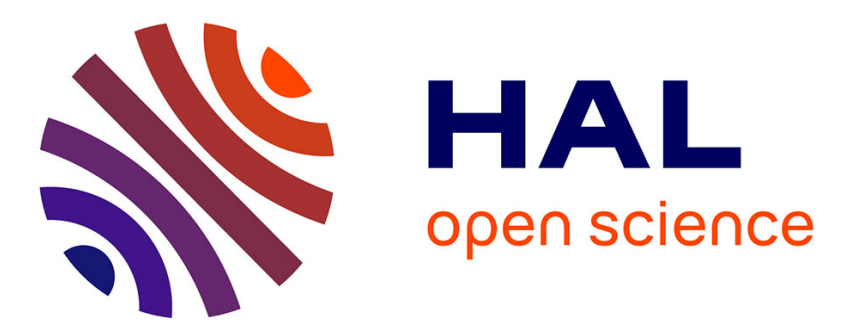

\title{
Multi-level tomography reconstructions with level-set and TV regularization methods
}

\author{
L. Wang, B Sixou, F Peyrin
}

\section{To cite this version:}

L. Wang, B Sixou, F Peyrin. Multi-level tomography reconstructions with level-set and TV regularization methods. 2016 24th European Signal Processing Conference (EUSIPCO), Aug 2016, Budapest, Hungary. pp.1698 - 1702, 10.1109/EUSIPCO.2016.7760538 . hal-01482142

\section{HAL Id: hal-01482142 \\ https://hal.science/hal-01482142}

Submitted on 3 Mar 2017

HAL is a multi-disciplinary open access archive for the deposit and dissemination of scientific research documents, whether they are published or not. The documents may come from teaching and research institutions in France or abroad, or from public or private research centers.
L'archive ouverte pluridisciplinaire $\mathbf{H A L}$, est destinée au dépôt et à la diffusion de documents scientifiques de niveau recherche, publiés ou non, émanant des établissements d'enseignement et de recherche français ou étrangers, des laboratoires publics ou privés. 


\title{
MULTI-LEVEL TOMOGRAPHY RECONSTRUCTIONS WITH LEVEL-SET AND TV REGULARIZATION METHODS
}

\author{
L.Wang, B.Sixou, F.Peyrin \\ * CREATIS, CNRS UMR 5220, Inserm U630, INSA de Lyon, Universite de Lyon, F-69621 \\ ${ }^{\dagger}$ ESRF,6 rue Jules Horovitz, F-38043, Grenoble Cedex France
}

\begin{abstract}
The discrete tomographic reconstruction problem is generally considered for binary image. In this work, we consider the reconstruction of an image with more than two grey levels and compare two reconstruction methods. The first one is based on a classical TV regularization and the second one is a levelset regularization method. In this second method, the discrete tomographic problem is formulated as a shape optimization problem with several level-set functions and regularized with Total Variation-Sobolev terms. The two methods are applied to an image size of $128 \times 128$, with several additive Gaussian noises on the raw projection data and several number of projections.
\end{abstract}

Index Terms - X-ray imaging, TV regularization, discrete tomography, level-set regularization, inverse problems.

\section{INTRODUCTION}

The tomographic reconstruction problem has many applications in medical imaging and material science. It is a highly ill-posed problem when the number of projection angles is decreased as it is the case for in vivo imaging of 3D bone micro-architecture [1] where the radiation dose has to be reduced [2]. Many attempts can be found in the literature to find stable solutions by various regularization methods like Total Variation (TV) or sparsifying transforms [3-8]. A very useful way to convert the few view tomographic reconstruction problem to a simpler problem is to consider it as a discrete inverse problem in which the function to be reconstructed can take a finite number of discrete values [9]. The most usual discrete tomography problem is the binary tomography problem which is associated with an under-determined linear system of equations with the linear Radon projection operator $R$ and binary constraints:

$$
R f=p^{\delta} \quad f=\left(f_{1}, \ldots \ldots f_{n}\right) \in\{0,1\}^{n}
$$

relating the pixel values $\left(f_{i}\right)_{1 \leq i \leq n}$ of the image and the measured projection values $p^{\delta}$ which is some approximation of the correct data $p$, corresponding to the true solution $f^{*}$ with $R f^{*}=p$. This binary approach is not sufficient in the case of multi-materials objects where the function to be reconstructed can take more than two different values. Many attempts can be found in the literature to solve the binary problem based on algebraic methods $[10,11]$ or convex analysis [12-14]. Markov random field can also be very useful [15]. Yet, there are few studies of the multi-level tomographic reconstruction problem. In a variational framework, the image is obtained by the minimizatin of a regularization functional $E(f)$ constructed with a data fidelity term that measures the consistency between the estimate and the measurements and a regularization term $J(f)$ that imposes an a priori constraint on the solution. The data-fitting term is usually based on the $L_{2}$ norm and the regularization functional can then be written as:

$$
E(f)=\left\|R f-p^{\delta}\right\|_{L_{2}}^{2}+\lambda J(f)
$$

The parameter $\lambda$ is the regularization parameter balancing the contribution of the two terms. The noisy data $p^{\delta}$ are corrupted by noise with a noise of level $\delta$, satisfying $\left\|p^{\delta}-p\right\|_{2} \leq \delta$. Recently, the binary tomography problem has been investigated with a comparison of the Total Variation and Level-set algorithm [16-18]. For an image $f \in H_{1}(\Omega)$, the TV regularization is based on computing the $L_{1}$ norm of the gradient $[19,20]$.

$$
J_{T V}(f)=\int_{\Omega}|\nabla f(r)| d r
$$

On the other hand, level-set methods, well established in the field of image processing, have been designed recently to reconstruct solutions of inverse problems with piecewise constant solutions [18,21-25].

The main contribution of this work is to investigate the level-set approach used for the binary tomography problem to the multi-level case and to compare this regularization method with the Total Variation approach. The comparison of the inversion schemes is performed on a simple Shepp-Logan phantom with several noise levels and different number of projections. This paper is structured as follows. After the introduction, the second section summarizes the Total Variation regularization method and the Alternate Direction of Minimization Method (ADMM) used to minimize the regularization functional. We also detail the nonlinear inverse problem formulation of the multi-level tomography problem together with the classical level-set regularization. The numerical results obtained are reported and discussed in the last section. Then main conclusions are given. 


\section{TOTAL VARIATION REGULARIZATION AND ADMM APPROACH}

For comparison purposes with the level-set regularization results, we have tried to solve the discrete tomography reconstruction problem with the Total Variation regularization. We have thus considered the following optimization problem:

$$
(P) \text { minimize } \frac{\mu}{2}\|p-R f\|_{2}^{2}+J_{T V}(f)
$$

A fast, efficient $T V / L_{2}$ minimization algorithm based on an augmented Lagrangian function and alternating minimization has been proposed recently $[19,20]$ and is now the state-ofart method to minimize the TV regularization functional. Our problem is thus formulated as a minimization problem of the ADMM form with the following Lagrangian function:

$$
\begin{aligned}
\mathcal{L}\left(f,\left(g_{i}\right),\left(\lambda_{i}\right)\right)= & \sum_{i}\left(\left\|g_{i}\right\|_{2}-\lambda_{i}^{t}\left(g_{i}-D_{i} f\right)\right. \\
& \left.+\frac{\beta}{2}\left\|g_{i}-D_{i} f\right\|_{2}^{2}\right)+\frac{\mu}{2}\left\|p^{\delta}-R f\right\|_{2}^{2}
\end{aligned}
$$

where $\mu$ is the regularization parameter and $\beta$ the Lagrangian parameter. The Lagrange multipliers $\left(\lambda_{i}\right)$ are vectors in $\mathbb{R}^{2 n^{2}}$. For each pixel $i, D_{i} f \in \mathbb{R}^{2}$ represents the first-order finite difference at pixel $i$ in both horizontal and vertical directions. The ADMM algorithm searches for the saddle point of the augmented Lagrangian by the following minimizations:

$$
\begin{gathered}
g_{i}^{k+1}=\arg \min _{g_{i}} \mathcal{L}\left(f^{k},\left(g_{i}\right),\left(\lambda_{i}^{k}\right)\right) \\
f^{k+1}=\arg \min _{f} \mathcal{L}\left(f,\left(g_{i}^{k+1}\right),\left(\lambda_{i}^{k}\right)\right) \\
\lambda_{i}^{k+1}=\arg \max _{\lambda_{i}} \mathcal{L}\left(f^{k+1},\left(g_{i}^{k+1}\right),\left(\lambda_{i}\right)\right)
\end{gathered}
$$

The sequences $\left(f^{k}, g_{i}^{k}, \lambda_{i}^{k}\right)$ generated by the ADMM algorithm converges to a Kuhn-Tucker point of problem (P), $\left(f^{*}, g_{i}^{*}, \lambda_{i}^{*}\right)$. The new iterate $f^{k+1}$ is obtained from the following linear system:

$$
\left(\sum_{i} D_{i}^{t} D_{i}+\frac{\mu}{\beta} R^{t} R\right) f^{k+1}=\sum_{i} D_{i}^{t}\left(g_{i}^{k+1}-\frac{1}{\beta} \lambda_{i}^{k}\right)+\frac{\mu}{\beta} R^{t} p^{\delta}
$$

\section{LEVEL-SET REGULARIZATION FOR MULTI-LEVEL TOMOGRAPHY}

\subsection{Level-set formulation of the multi-level tomography prob- lem}

Our new level-set tomography method is based on a formulation of the reconstruction as a nonlinear inverse problem [26]. For $\Omega$ a bounded Lipschitz open subset in $\mathbb{R}^{2}$, we assume that the function to be reconstructed $f$ can take different values $\left(f_{i}\right)$ on regular sets $\Omega_{i} \subset \Omega$, and the function $f$ can thus be written $f=\sum_{i} \chi_{\Omega_{i}}$. In the following, for the sake of simplicity, we consider in this work only the case of three levels for the function to reconstruct, $f_{1}, f_{2}$ and $f_{3}$. We assume that the image $f$ to be reconstructed can be represented with two level-set functions $\theta_{1}$ and $\theta_{2}$ :

$$
\begin{array}{r}
f=f_{1}\left(1-H\left(\theta_{1}\right)\right)\left(1-H\left(\theta_{2}\right)\right)+f_{2} H\left(\theta_{1}\right) H\left(\theta_{2}\right) \\
+f_{3} H\left(\theta_{2}\right)\left(1-H\left(\theta_{1}\right)\right)
\end{array}
$$

where the two level-set functions $\theta_{1}$ and $\theta_{2}$ belongs to the firstorder Sobolev space $H_{1}(\Omega)$. The Heaviside distribution is defined by $H(\theta)=1$ if $\theta>0$ and 0 otherwise.

With respect to $\theta_{1}$ and $\theta_{2}$, the reconstruction problem becomes nonlinear and consists in determining the level-set functions $\theta_{1}$ and $\theta_{2}$ such that $R H\left(\theta_{1}, \theta_{2}\right)=p^{\delta}$. Since $H$ is discontinuous, it is necessary to consider generalized minimizers of the regularization functional [21,22]. These minimizers can be approximated by minimizers of smoothed regularization functional with an approximation function $H_{\epsilon}$. The regularization functional to be minimized can then be written as:

$$
E\left(\theta_{1}, \theta_{2}\right)=\frac{\left\|R H\left(\theta_{1}, \theta_{2}\right)-p^{\delta}\right\|_{2}^{2}}{2}+\alpha\left(F\left(\theta_{1}\right)+F\left(\theta_{2}\right)\right)
$$

where $F$ is a regularization term for the level-set functions. In this work, we considered a Total Variation- $H_{1}$ regularization functional [21,22] for each level-set function:

$$
F(\theta)=\beta_{1} \int|\nabla H(\theta)| d x+\beta_{2}\|\theta\|_{H_{1}}^{2}
$$

The regularization parameters $\beta_{1}, \beta_{2}$ determines the relative weights of the stabilizing terms. For the sake of simplicity, they were taken equal for the two level-set functions $\theta_{1}$ and $\theta_{2}$.

\subsection{Implementation of the level-set regularization approach}

In the numerical implementation, it is necessary to replace to Heaviside function $H$ and the Dirac function $\delta$ by smoothed approximations. The following smooth approximations of the Heaviside function $H$ has been used $H_{\epsilon}(x)=\frac{1+2 \epsilon}{2}(\operatorname{erf}(x / \epsilon)+1)-\epsilon$, where $\epsilon$ is a real positive constant. The smoothing parameters for $\epsilon_{1}$ and $\epsilon_{2}$ are given the same value in this work.

The smoothed Tikhonov regularization functional is then given by:

$$
\begin{aligned}
& E_{\epsilon}(\theta)= \frac{\left\|R f\left(\theta_{1}, \theta_{2}\right)-p^{\delta}\right\|_{2}^{2}}{2}+\beta_{1}\left|H_{\epsilon}\left(\theta_{1}\right)\right|_{T V}+ \\
& \beta_{2}\left\|\theta_{1}\right\|_{H_{1}}^{2}+\gamma_{1}\left|H_{\epsilon}\left(\theta_{2}\right)\right|_{T V}+\gamma_{2}\left\|\theta_{2}\right\|_{H_{1}}^{2}
\end{aligned}
$$

where $|\cdot|_{T V}$ is the Total Variation semi-norm. The minimizers of the Tikhonov functionals are found with a first-order optimality condition for the two level-set functions for the smoothed functionals, $\frac{\partial E_{\epsilon}}{\partial \theta_{1}}=G_{1}\left(\theta_{1}, \theta_{2}\right)=0$ and similarly $G_{2}\left(\theta_{1}, \theta_{2}\right)=0$, with :

$$
\begin{array}{r}
G_{1}\left(\theta_{1}, \theta_{2}\right)=\frac{\partial f}{\partial \theta_{1}} R^{*}\left(R f\left(\theta_{1}, \theta_{2}\right)-p^{\delta}\right) \\
+\beta_{2}(I-\Delta)\left(\theta_{1}\right) \\
+\beta_{1} \frac{\partial\left|H_{\epsilon}\left(\theta_{1}\right)\right| T V}{\partial \theta_{1}}
\end{array}
$$

where $R^{*}$ denotes the adjoint of the forward projection operator. The derivatives of $f$ with respect to $\theta_{1}$ and $\theta_{2}$ can be written:

$$
\begin{aligned}
\frac{\partial f}{\partial \theta_{1}}=\left(f_{2}-f_{3}\right) H_{\epsilon}\left(\theta_{2}\right) H_{\epsilon}^{\prime}\left(\theta_{1}\right)-f_{1} H_{\epsilon}^{\prime}\left(\theta_{1}\right)\left(1-H_{\epsilon}\left(\theta_{2}\right)\right) \\
\frac{\partial f}{\partial \theta_{2}}=f_{2} H_{\epsilon}^{\prime}\left(\theta_{2}\right) H_{\epsilon}\left(\theta_{1}\right)+f_{3} H_{\epsilon}^{\prime}\left(\theta_{2}\right)\left(1-H_{\epsilon}\left(\theta_{1}\right)\right) \\
-f_{1}\left(1-H_{\epsilon}\left(\theta_{1}\right)\right) H_{\epsilon}^{\prime}\left(\theta_{2}\right)
\end{aligned}
$$

where the derivative $H_{\epsilon}^{\prime}$ is the smoothed approximation of the Heaviside function and the multiplication is to be understood pixelwise. 
The differential of the $\left|H_{\epsilon}\left(\theta_{1}\right)\right|_{T V}$ is given by:

$$
\frac{\partial\left|H_{\epsilon}\left(\theta_{1}\right)\right|_{T V}}{\partial \theta_{1}}=-\delta\left(\theta_{1}^{k}\right) \frac{\nabla \theta_{1}}{\left|\nabla \theta_{1}\right|}
$$

From the current estimate $\theta_{1}^{k}$, the update $\theta_{1}^{k+1}=\theta_{1}^{k}+\delta \theta$ is obtained with the variation $\delta \theta$ which is calculated with a Gauss-Newton method with a linearization of the condition $G_{1}\left(\theta_{1}^{k}+\delta \theta\right)=0$ [26]:

$$
V_{k}^{*} V_{k} \delta \theta+\beta_{2}(I-\Delta)(\delta \theta)-\beta_{1} \delta\left(\theta_{1}^{k}\right) \nabla \cdot \frac{\nabla \delta \theta}{\left|\nabla \theta_{1}^{k}\right|}=-G\left(\theta_{1}^{k}\right)
$$

where $V_{k}$ is the operator $V_{k}=R \frac{\partial f}{\partial \theta_{1}}\left(\theta_{1}^{k}\right)$. And the same formula holds for the level-set function $\theta_{2}$. These symmetric linear systems are solved alternatively by a conjugate gradient method.

\section{RESULTS AND DISCUSSION}

In this section, we present the simulation details and the results obtained with the Total Variation and level-set regularization.

\subsection{Simulation details}

The TV and level-set regularization methods have been compared on a three grey levels Shepp-Logan phantom displayed in Fig. 1 of size $128 \times 128$, which is reconstructed from $N_{p}=400$ projections with $N_{r}=185 \mathrm{X}$-rays per projection with Filtered Back Projections (FBP). In our simulation, this image regarded as the "ground-truth" image. This reference image has the values $f_{1}=0$ (blue region), $f_{1}=1$ (green region) and $f_{2}=2$ (red region).

The reconstruction methods were applied to the raw projection data $p$ with Gaussian noises with various standard deviation $\sigma_{p}$. The noise level $\delta$ of the noisy projection data $p^{\delta}$ is given by $\delta=\sqrt{M N_{p}} \sigma_{p}$. For comparison, the FBP algorithm has also been tested on the same phantom with the same noise levels and number of projections. In our experiments, the projection operator $R$ is taken as the discrete approximation of Radon transform, which is implemented in Matlab Toolbox. Two kinds of Gaussian noises have been added to the raw projection data $p$ with the standard deviations $\sigma_{p}=3$ and $\sigma_{p}=6.5$ corresponding to Peak to Peak Signal to Noise Ratio (PPSNR) of $18 d B$ and $12 d B$. The TV and level-set regularization methods were tested on a limited number of views, $M$, with $M=20,30$ or 50 .

In our simulations, based on the Morozov principle [27], the TV regularization parameter $\mu$ was chose when it satisfies the condition: $\frac{||\left|R f(\mu)-p^{\delta} \|-\delta\right|}{\delta} \leq 0.1$, where $f(\mu)$ is the reconstructed image obtained at the end of TV process with the regularization parameter $\mu$. The ADMM iterations are stopped when the iterates stagnate $\frac{\left\|f^{k+1}-f^{k}\right\|}{\left\|f^{k}\right\|} \leq 0.01$.

In the level-set approach, the initial level-set functions were set to $\theta_{1}=0$, and $\theta_{2}=0$. The real positive constant $\epsilon$ controlling the scale of the smoothed Dirac function was set as 11 . Better reconstruction results are obtained when $\epsilon$ is gradually decreased. With this decrease, the convergence speed becomes lower. The level-set regularization parameters of $\beta_{1}$ and $\gamma_{1}$ were set to 0 because the $H_{1}$ term dominates the TV term $[21,22]$. The regularization parameters are chosen to obtain the best decrease of the discrepancy term. The image obtained for the minimum value of the discrepancy term is considered as the best reconstructed image. At the end of the two reconstruction processes, the grey-level reconstructed images were projected on the discrete values: $f=\{0,1,2\}$ with thresholds 0.5 and 1.3 .

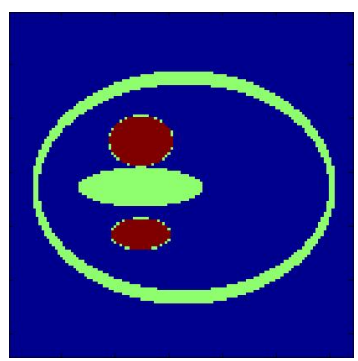

Fig. 1. Reconstruction of the bone cross-section from 400 projections with the FDK algorithm, as the "ground-truth" image.

\subsection{Numerical results}

As an example of the reconstruction results obtained, the grey-level and discrete reconstructed images with $M=50$ projection angles, for the standard deviation $\sigma_{p}=3$, are shown in Fig.2 and Fig.3 respectively for TV regularization and the Level-set method for the noise level $\sigma_{P}=3$. The difference maps corresponding to Fig.3 are displayed in Fig.4. In both cases, the reconstructed errors are localized on the boundaries.

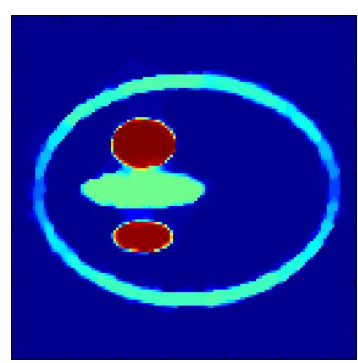

(a)

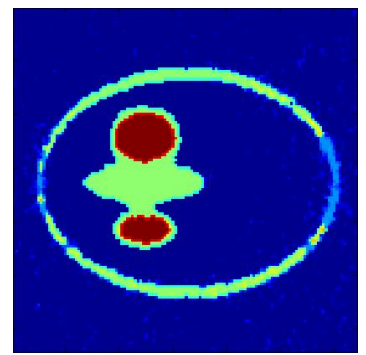

(b)
Fig. 2. Grey-level reconstructed images with 50 projections for $\sigma_{p}=3$ (a) TV; (b) LS

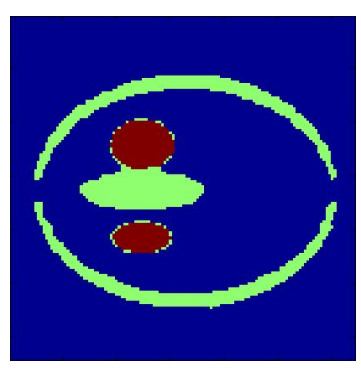

(a)

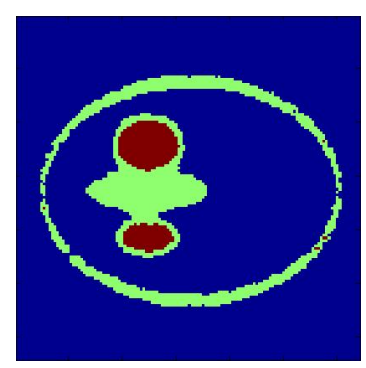

(b)
Fig. 3. Discrete reconstructed images with 50 projections for $\sigma_{p}=3$ (a) TV; (b) LS 

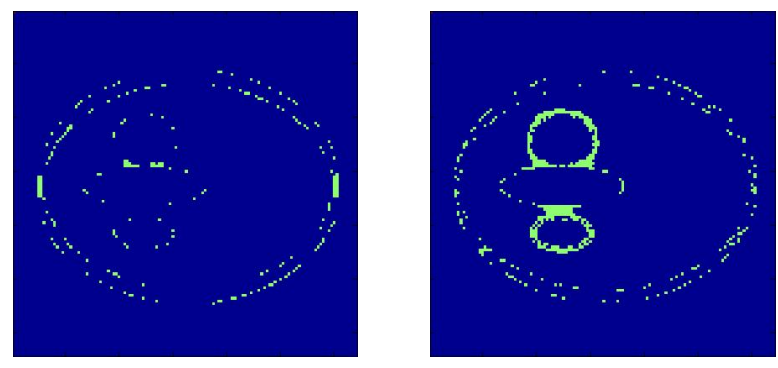

(a)

(b)

Fig. 4. Difference maps between the ground-truth and the reconstructed images with 50 projection for $\sigma_{p}=3$. (a) TV; (b) LS

In order to evaluate the quality of reconstruction, we have calculated the evolution of the discrepancy term $\left\|R f^{k}-p^{\delta}\right\|$ and of the missclassification rate $M R$ as a function of the number of iterations $k$. The misclassification rate $M R$ was defined to estimate the error between the final discrete images $f_{b}$ and the ground-truth $f^{*}$. It is defined as:

$$
M R=\frac{N_{d}}{N} \times 100 \%
$$

where $N_{d}$ is the number of different pixels in the discrete reconstructed image and ground-truth image, and $N$ is the total number of pixels.

The evolution curves of the discrepancy term and of the misclassification rate are displayed in Fig.5 and Fig.6, for the different number of projections investigated for the noise level $\sigma_{p}=3$ for the two regularization approaches.

The missclassification rates obtained with the Total Variation, level-set and FBP algorithm are summarized in Table.1 for the various noise levels and number of projections. In most cases, the minimum reconstruction errors are obtained with the TV regularization.

Table 1. Missclassification rates (\%) obtained with Total Variation, level-set and FBP approaches

\begin{tabular}{cccc}
\hline \hline & TV & LS & FBP \\
\hline \hline$\sigma_{p}=3, \mathbf{M}=20$ & 1.73 & 3.39 & 19.16 \\
$\sigma_{p}=3, \mathbf{M}=30$ & 1.40 & 2.88 & 13.47 \\
$\sigma_{p}=3, \mathbf{M}=50$ & 1.31 & 2.84 & 6.13 \\
$\sigma_{p}=6.5, \mathbf{M}=20$ & 2.87 & 6.98 & 34.64 \\
$\sigma_{p}=6.5, \mathbf{M}=30$ & 2.89 & 5.16 & 29.46 \\
$\sigma_{p}=6.5, \mathbf{M}=50$ & 1.90 & 3.58 & 23.13 \\
\hline
\end{tabular}

The reconstruction results obtained with the TV ADMM algorithm are better than the ones obtained with the level-set method. TV regularization method is well-known to preserve image edges and provide good reconstruction images with sparse view sampling. The level line of the Shepp-Logan phantom is well restored with the TV regularization term which tends to minimize its perimeter. The boundaries of the reconstructed images obtained with TV regularization are much smoother. A more complex object will be tested as before [18] in the future because the level-set method may be a more efficient method for objects with a complex topology structure.

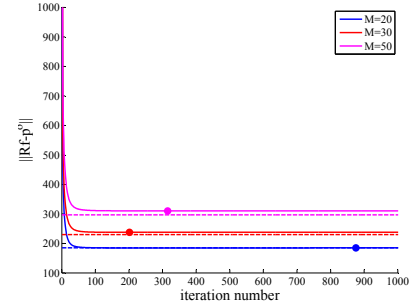

(a)

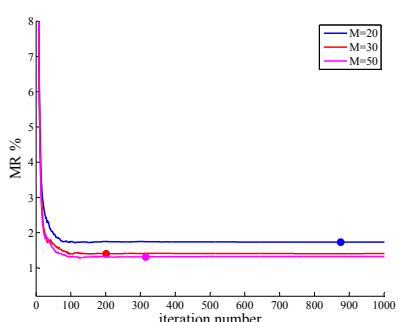

(b)
Fig. 5. (a) Evolution with the iterations of $\left\|R f-p^{\delta}\right\|$ for TV method. (b) Evolution with the iterations of the misclassification rate for TV method. We chose the best reconstruction according to the Morozov principle.

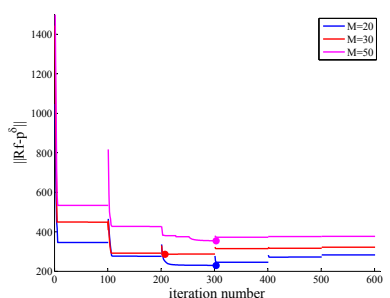

(a)

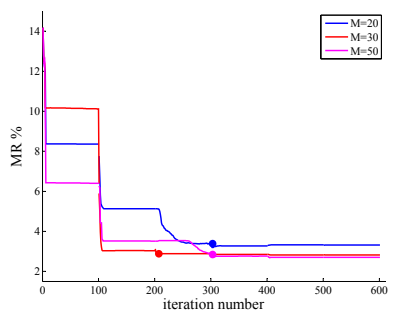

(b)
Fig. 6. (a) Evolution with the iterations of $\left\|R f-p^{\delta}\right\|$ for LS method. (b) Evolution with the iterations of the misclassification rate for LS method. We chose the best reconstruction with the minimum value of the discrepancy term.

\section{CONCLUSION}

In this paper, two reconstruction methods for multi-level discrete tomography with a limited number of projections have been compared. The first one is the classical TV regularization approach. The optimal solution is obtained with the ADMM algorithm. The second method is based on a representation of the function to be reconstructed with several level-set functions which leads to a non-linear inverse problem formulation of the discrete tomography problem. The simple tests done on the simple Shepp-Logan phantom show that the TV regularization outperforms the level-set methods on most cases. Experiments on more complex objects would be necessary to further evaluate the proposed level-set method. Other methods will be compared with these approaches on more complex objects in future studies.

\section{REFERENCES}

[1] M. L. Bouxsein, S. K. Boyd, B. A. Christiansen, R. E. Guldberg, K. J. Jepsen, and R. Mller. Guidelines for assessment of bone microstructure in rodents using micro-computed tomography. Journal of Bone and Mineral Research, 25:1468-1486, 2010.

[2] S. Boutroy, N. Vilayphiou, J. P. Roux, P. D. Delmas, H. Blain, R. D. Chapurlat, and P. Chavassieux. Comparaison of $2 \mathrm{~d}$ and $3 \mathrm{~d}$ bone microarchitecture evaluation at the femoral neck, 
among postmenopausal women with hip fracture or hip osteoarthritis. Bone, 49(5):1055-1061, 2011.

[3] G. H. Cheng, J. Tang, and S. Leng. Prior image constrained compressed sensing (piccs): A method to accurately reconstruct dynamic ct images from highly undersampled projection data sets. Medical Physics, 35, 2008.

[4] X.H. Duan, L. Zhang, and Y. X. Xing. Few-view projection reconstruction with an iterative reconstruction-reprojection algorithm and tv constraint. IEEE Transactions on Nuclear Science, 56:1377-1382, 2009.

[5] E. Y. Sidky and X. Pan. Accurate image reconstrution feom few-views and limited-angle data in divergent-beam CT. J.Xray Sci.Techn., 14:119-139, 2006.

[6] E. Y. Sidky and X. Pan. Image reconstruction in circular cone-beam computed tomography by constrained total variation minimization. Phys.Med.Biol., 53:4777-4807, 2008.

[7] L. Ritschl, F. Bergner, C. Fleischmann, and M. Kachelriess. Improved total variation-based CT image reconstruction applied to clinical data. Phys.Med.Biol., 56:1545-1561, 2011.

[8] H. Yu and G. Wang. A soft-threshold filtering approach for reconstruction from a limited number of projections. Phys.Med.Biol., 55:3905-3916, 2010.

[9] G. T. Herman and A. Kuba. Advances in discrete tomography and its applications. Applied and Numerical Harmonic Analysis. Birkhauser, Boston, MA, 2007.

[10] K. J. Batenburg and J. Sijbers. Generic iterative subset algorithm for discrete tomography. Discrete Applied Mathematics, 157:438-451, 2009.

[11] W. Cai and L. Ma. Comparaison of approaches based on optimization and algebraic iteration for binary tomography. Computer Physics Communications, 181:1974-1981, 2010.

[12] T.D. Capricelli and P.L. Combettes. Advances in discrete tomography and its applications: A convex programming algorithm for noisy discrete tomography. Birkhauser, Boston, MA, 2007.

[13] T. Schlea, C. Schnrra, S. Webera, and J. Horneggerb. Discrete tomography by convex-concave regularization and D.C programming. Discrete Applied Mathematics, 151:229-243, 2005.

[14] B. Sixou and F. Peyrin. Reconstruction of bone microstructure from few projections with convex-concave and non local regularization. In Biomedical Imaging (ISBI), pages 1443-1446, Barcelona, Spain, May 2012.

[15] H. Y. Liao and G.T. Herman. Automated estimation of the parameters of the Gibbs priors to be uses in binary tomography. Discrete Applied Mathematics, pages 249-170, 2004.

[16] L. Wang, B. Sixou, and F. Peyrin. Binary tomography reconstructions of bone microstructure from few projections with stochastic level-set methods. In Image Processing (ICIP), pages 1778-1782, Paris,France, October 2014.

[17] L. Wang, B. Sixou, and F. Peyrin. Binary tomography reconstructions with stochastic level-set methods. IEEE Signal Processing Letters, 22(7):920-924, 2015.
[18] L. Wang, B. Sixou, S. Rit, and F. Peyrin. Binary tomography reconstruction from few projection with level-set regularization methods for bone microstructure study. International Journal of Tomography and Simulation, 29(1), 2016.

[19] M. K. Ng, P. Weiss, and X. Yuan. Solving constrained totalvariation image restoration and recontruction problems via alternating direction methods. SIAM Journal on Scientific computing, 32:2710-2736, 2010.

[20] W.Yin Y.Wang, J.Yang and Y.Zhang. A new alternating minimization algorithm for total variation image reconstruction. SIAM J.Imaging Sciences, 1:248-272, 2008.

[21] A. Egger and L. Leitao. Nonlinear regularization for ill-posed problems with piecewise constant or strongly varying solutions. Inverse Problems, 25(115014), 2009.

[22] A. DeCezaro, A. Leitao, and X. C. Tai. On multiple level-set regularization methods for inverse problems. Inverse Problems, 25(035004), 2009.

[23] X. C. Tai and T.F. Chan. A survey on multiple level-set methods with applications for identifying piecewise constant functions. International Journal of Numerical Analysis and Modeling, 1:25-47, 2004.

[24] F. Fruhauf, O. Scherzer, and A. Leitao. Analysis of regularization methods for the solution of ill-posed problems involving discontinuous operators. SIAM Journal onNumerical Analysis, 43:767-786, 2005.

[25] A. DeCezaro, A. Leitao, and X. C. Tai. On piecewise constant level-set (PCLS) methods for the identification of discontinuous parameters in ill-posed problems. Inverse Problems, 29(015003), 2013.

[26] B Sixou, L Wang, and F Peyrin. Binary tomographic reconstruction of bone microstructure from few projections with level-set regularization. In IEEE Symposium on Biomedical Imaging, San Francisco, 2013.

[27] V. A. Morozov. Methods for solving incorrectly posed problems. Springer-Verlag, Berlin-Heidelberg-New York-Toky, 1984. 